\title{
Digital Fabrication: an Outlook from Digital Tectonics
}

\author{
Vianey Eunice Sánchez Velasco \\ Maestría en Arquitectura, Universidad Veracruzana | México | via.archit@gmail.com
}

Selim Abdel Castro Salgado

Universidad Veracruzana | México | selim_castro@hotmail.com

\begin{abstract}
This work seeks to identify the relationships between digital manufacturing technologies and the different views about the tectonic component of architecture. To accomplish so, we have reviewed recent research that encompasses these concepts and their processes and methodologies of project design, instrumental and theoretical development, through which an analysis of these two aspects of current architectural practice can be identified and proposed.
\end{abstract}

Keywords: Digital Tectonics; Digital Fabrication; Research.

\section{INTRODUCCIÓN}

Lo tectónico engloba la dimensión material de la arquitectura. A pesar de que este término ha tenido una amplia evolución a lo largo de los últimos siglos, la etimología de la palabra, tektonikós, refiriere a la construcción o la habilidad en construir: "1. adj. Designa lo perteneciente o relativo a los edificios u otras obras de arquitectura" (Real Academia, 2001). Este término también ha sido definido, discutido y estudiado por diversos teóricos de la arquitectura a través de la historia, desde Semper (1803-1879) que lo define como un fenómeno cultural que determina como se utilizan los materiales en la arquitectura (Oxman R. , 2009, pág. 940) a Seckler (1965), retomado a finales del siglo XX por Frampton (1999), y determinado como el potencial de la realización precisa, la instrumentalidad, el esmero y el cuidado de la materia formada, con la expresión de los materiales que constituyen no solo una habilidad técnica, sino la lógica constructiva que tiene su mediación entre la tecnología, los modos productivos y la intencionalidad.

En el siglo XXI, la influencia de las tecnologías e instrumentalidades digitales empleadas en la arquitectura ha resultado en muy variados debates. Picon (2004) plantea que el diseño asistido por la computadora tiende a descuidar la dimensión material de la arquitectura, así como su relación con las propiedades físicas del peso, los esfuerzos mecánicos, la resistencia, y las restricciones propias de los materiales, debido a que los modelos virtuales parecen "flotar", descuidado la dimensión material de la arquitectura. Andersson y Kirkegaard (2006) exponen que el término tectónicas digitales podría parecer contradictorio, ya que lo digital nos remite a un pensamiento sobre lo virtual, abstracto y libre de las leyes de la naturaleza, mientas que lo tectónico se relaciona con lo material, lo táctil, lo que se encuentra sujeto a las leyes de la naturaleza. Y es este el punto decisivo de la nueva concepción de la tekne.

En la integración de la tekne digital, el concepto de lo tectónico en la arquitectura gira hacia un sentido de integración de los materiales como base de diseño (Oxman, (2009) (2012). Así se entiende que el término "tectónicas digitales" se presenta en recientes investigaciones, instrumentales y teóricas, que buscan relacionar el escenario contemporáneo de producciones digitales con los aspectos tectónicos, encontrando un reciente aporte en Schumacher (2017) y su tectonicismo.

El propósito de análisis de este trabajo y de los artículos elegidos fue encontrar el planteamiento que acompaña al concepto tectónico propuesto en las investigaciones que igualmente retoman prácticas de fabricación digital. Esto con la intención de identificar cómo se emplea el concepto en el desarrollo de las prácticas, principalmente, instrumentales. Asimismo, hemos indagado en los análisis y estudios de casos que permiten conocer los problemas, perspectiva teórica, características de los métodos y herramientas de diseño (con sus respectivas condicionantes y restricciones) y los principales resultados de estas prácticas de fabricación digital. Debido a que el concepto de lo tectónico se ha encontrado en continuo desarrollo y redefinición como uno de los conceptos clave que pone de relevancia los aspectos socioculturales y sus contextos de práctica, en la actualidad, podemos plantearnos analizarlo desde el enfoque de naturaleza disruptiva de la fabricación digital en la práctica de la arquitectura (Veliz Reyes, y otros, 2019, pág. 9).

La numeración que se presenta en los diferentes apartados corresponde solo al deseo de diferenciar entre los distintos enfoques y artículos retomados en este trabajo, sin que esto signifique su correspondencia por número. Para evitar confusiones, se agrega la cita al final de cada apartado buscando facilitar la identificación del artículo y los aspectos importantes de acuerdo con cada lector.

\section{TECTÓNICAS DE FABRICACIÓN DIGITAL: DEFINICIÓN DE PARÁMETROS Y CARACTERÍSTICAS DE LA MUESTRA DOCUMENTAL}

Para el desarrollo de este trabajo se buscaron, recolectaron, analizaron y eligieron diferentes artículos científicos y tesis doctorales que retoman las investigaciones de los últimos años que muestran la relación que guardan los conceptos: tectónica, construcción, diseño y fabricación digital, y otros afines. Se buscó obtener certeza de la pertinencia, métodos y diseño de investigación que han permitido desarrollar estas 
investigaciones en los últimos cinco años, a nivel internacional. Se obtuvieron artículos en español, inglés y portugués, mediante los cuales, se pretende tender un panorama que permita analizar sus argumentos, tendencias y perspectivas metodológicas.

\section{TABLA DE ARTÍCULOS SELECCIONADOS}

Tabla 1: Listado de artículos seleccionados y analizados para este trabajo

\begin{tabular}{|c|c|c|}
\hline Autor & Año & Título \\
\hline $\begin{array}{l}\text { Soungmin Yu; } \\
\text { Soomeen } \\
\text { Hahma; Shajay } \\
\text { Bhooshana }\end{array}$ & 2014 & $\begin{array}{l}\text { Performance driven self- } \\
\text { supporting curved surface } \\
\text { morphologies and tectonic in } \\
\text { current practice }\end{array}$ \\
\hline $\begin{array}{l}\text { W.X. Huang; D. } \\
\text { Yan; P. Luo; X.L. } \\
\text { Li }\end{array}$ & 2016 & $\begin{array}{l}\text { Digital design and construction of } \\
\text { a weaving structure }\end{array}$ \\
\hline $\begin{array}{l}\text { Baliński } \\
\text { Grzegorz; } \\
\text { Januszkiewics } \\
\text { Krystyna }\end{array}$ & 2016 & $\begin{array}{l}\text { Digital Tectonic Design as a New } \\
\text { Approach to Architectural Design } \\
\text { Methodology }\end{array}$ \\
\hline Michał, Golański & 2018 & $\begin{array}{l}\text { Digital tectonics and dynamics in } \\
\text { designing of wooden architecture } \\
\text { envelopes }\end{array}$ \\
\hline $\begin{array}{l}\text { Indrawan } \\
\text { Stephanus } \\
\text { Evert; Purwoko } \\
\text { Gervasius Herry; } \\
\text { Noviyanto Tri }\end{array}$ & 2019 & $\begin{array}{l}\text { Reparametrizing Tectonics } \\
\text { Perception on Planar Material- } \\
\text { Design }\end{array}$ \\
\hline $\begin{array}{l}\text { Caio Augusto } \\
\text { Rabite de } \\
\text { Almeida; } \\
\text { Fernando Tadeu } \\
\text { de Araújo Lima; } \\
\text { Marcos Martins } \\
\text { Borges }\end{array}$ & 2019 & $\begin{array}{l}\text { Tectônicas Digitais: a } \text { (in) } \\
\text { tangibilidade no processo de } \\
\text { projeto em arquitetura }\end{array}$ \\
\hline $\begin{array}{l}\text { Katie } \\
\text { MacDonald, } \\
\text { Jonas } \\
\text { Hauptman, Kyle } \\
\text { Schumann }\end{array}$ & 2019 & $\begin{array}{l}\text { Digital Fabrication of Standard } \\
\text { less Materials }\end{array}$ \\
\hline $\begin{array}{l}\text { Alejandro Veliz } \\
\text { Reyes, Wassim } \\
\text { Jabi, Mohamed } \\
\text { Gomaa, } \\
\text { Aikaterini } \\
\text { Chatzivasileiadi, } \\
\text { Lina Ahmad y } \\
\text { Nicholas Mario } \\
\text { Wardhana }\end{array}$ & 2019 & $\begin{array}{l}\text { Negotiated matter: a robotic } \\
\text { exploration of craft driven } \\
\text { innovation }\end{array}$ \\
\hline
\end{tabular}

\section{PROBLEMAS DE INVESTIGACIÓN}

Los problemas de investigación planteados se relacionan con:

1. La falta de procesos de proyecto que permitan integrar los aspectos tectónicos y constructivos con los aspectos de proyecto arquitectónico e ingeniería estructural (Huang, Yan, Luo, \& Li, 2016).

2. El reconocimiento de las posibilidades de fusionar el diseño y la fabricación digital en la generación de elementos que relacionen las características de los materiales con las restricciones de fuerza y sus capacidades estructurales; específicamente en el diseño de estructuras con madera laminada (Golański, 2018).

3. La comprobación de que la integración de diseño computacional y las herramientas digitales posibilitan la integración del material, estructura, y la forma asociada con la fabricación y los modelos planteados (Indrawan, Purwoko, \& P. Utomo, 2019).
4. El abordaje de procesos de diseño paramétrico computacional, con variables y parámetros digitales y constructivos (Rabite De Almeida, Lima, \& Martins Borges, 2019).

5. El reconocimiento de un paradigma "emergente" en la tectónica digital, donde el proyecto arquitectónico se ve renovado por el interés en la producción industrial, lo que requiere nuevas herramientas y pensamientos de proyecto enfocados hacia lo constructivo (Baliński \& Januszkiewics, 2016).

6. Las formas y métodos de fabricación interdisciplinares, que utiliza metodología computacional para integrar de principio a fin las restricciones reales de la industria de la construcción en el proceso de proyecto (Yu, Hahm, \& Bhooshan, 2014).

7. Los métodos integrales de fabricación digital que analiza los materiales, optimiza piezas dadas, ensambla, ajusta mediante diseño y retroalimentación paramétrica, entre el material disponible y el diseño del conjunto que permite ajustes de la geometría de acuerdo a las piezas disponibles (MacDonald, Hauptman, \& Schumann, 2019).

8. La contrastación del concepto de artesanía como un proceso abierto de descubrimiento y negociación, que implica propiedades materiales, modos divergentes de producción y representación del conocimiento, relacionada con la fabricación robótica presentada como altamente controlada y en donde las expectativas de resultados se anticipan, simulan, visualizan y corrigen antes de comenzar un proceso de producción. De esta manera, se presentan configuraciones tectónicas emergentes e interacción incorporada con la tecnología, mediando la interacción con el material y el uso de tecnologías robóticas, en un contexto de las disciplinas artesanales (Veliz Reyes, y otros, 2019).

\section{APROXIMACIONES METODOLÓGICAS DE LAS INVESTIGACIONES}

Las metodologías de diseño de las investigaciones, se pueden dividir en dos grupos, una en la que se desarrollan casos prácticos: en los cuales se exponen y aplican, diversos procesos y metodologías para diseño y fabricación digital; y por otro lado, se puede reconocer metodologías cualitativas donde por medio de análisis de casos de estudio, los procesos y metodologías de diseño y fabricación se logra un análisis de los distintos trabajos de despachos de arquitectura como el caso de Zaha Hadid, o casos específicos como el análisis de estructuras de madera laminar u objetos de fabricación aditiva.

\section{ENFOQUES EPISTEMOLÓGICOS PREDOMINANTES}

En las investigaciones planteadas en los artículos, se puede reconocer una constante de teorías que las sustentan. Se identifican aquellas teorías que se enfocan a los cambios o giros que ha dado la arquitectura con la inserción de las computadoras, el cambio al pensamiento computacional, de diseño y modelado, así como las diversas ideas del cambio de los procesos de diseño y las búsquedas de forma basadas en los materiales y en los diversos tipos de fabricación (autores específicos para cada tipo de fabricación). Otro aspecto que se puede identificar es el cambio de la comprensión del término tectónico y su evolución a lo que se nos presenta actualmente como tectónicas digitales, que confluye en diversos aspectos interdisciplinares de arquitectura, 
ingeniería y construcción. Entre los principales teóricos que se repiten en la mayoría de los casos, podemos encontrar a: Keneth Frampton (1999), Anderson \& Kinkergaard (2006), Liu (2006), Branko Kolarevic (2009), Lisa Iwamoto (2009), Rivka Oxman (2009) (2012), Neri Oxman y Rosenberg (2007), y Patrik Schumacher (2017), entre otros. En el caso del articulo realizado en China, se evidencia la diferencia de autores, $y$, al realizar una búsqueda de estos referentes teóricos, los libros o artículos no se encontraron y los que se lograron localizar no están traducidos al inglés.

\section{METODOLOGÍAS DESARROLLADAS: DE DISEÑO Y FABRICACIONES DIGITALES}

Las metodologías implementadas en las investigaciones fueron las siguientes:

1. Desarrollo de modelo 3D, geometría general, posteriormente convertida en un tejido con varilla, simulación estructural utilizando Rhinoceros Grasshopper y plataforma Kangaroo. Estudio de uniones y conjunto, dibujos de construcción que permitieron optimizar el flujo de trabajo y definir el proceso de construcción (Huang, Yan, Luo, \& Li, 2016).

2. Análisis de comportamiento de diseño es asociativo, manejo y modificación de la relación entre los objetos, proceso generativo, mostrando relaciones numéricas o gráficas. Seccionamiento, corte CNC y armado (Indrawan, Purwoko, \& P. Utomo, 2019).

3. Modelado de pabellón, desarrollo de la forma con Rhino y Grasshopper, posteriormente en el plug-in Scan-and-Solve, desarrollo de la lógica constructiva, análisis de la estructura, las áreas de esfuerzos y flexión de acuerdo al tipo de material, definición, despiece de la estructura para la fabricación y ejecución utilizando una máquina de corte CNC (Rabite De Almeida, Lima, \& Martins Borges, 2019).

4. Método de fabricación digital que analizas piezas disponibles de bambú, diseño de ensambles, ajustando los elementos por medio de diseño y retroalimentación paramétrica, con evaluación, optimización, fresado y desarrollo de uniones de bambú. Empleando un proceso análogo, midieron, marcaron, fotografiaron y posteriormente a través de un sistema de escaneo 3D y análisis fotográfico de los elementos de bambú, desarrollando uniones ideales, y mediante CNC desarrollo de componentes estructurales de bambú a escala 1:1 (MacDonald, Hauptman, \& Schumann, 2019).

5. Determinación sistema de impresión 3D "cob" (barro) con soporte robótico y sistemas de extrusión, estudios y diseño y experimentación de materiales, análisis cuantitativos de las características del material; pruebas de viabilidad de una pared impresa en 3D a escala real. Uso de software Grasshopper (McNeel Rhinoceros, para el modelado con Building Information Modelling (Autodesk Revit R_) y la simulación, optimización de la forma y la tensión estructural con (Autodesk Fusion360 R_) (Veliz Reyes, y otros, 2019)

6. Metodologías de análisis: descripción y análisis mediante casos de estudio, de las técnicas de modelado de polígonos, metodología de trabajo, así como las herramientas personalizadas desarrolladas por el grupo de investigación computacional CODE de ZHA. En los casos se describe el proceso para encontrar la forma, posteriormente proceso y software de modelado o simulación en 3D, solución de manera óptima de la estructura que genera una estética correcta, desarrollando el diseño ideal para su producción o construcción (Yu, Hahm, \& Bhooshan, 2014).

7. Análisis de casos de estudio, considerando: lógica constructiva de las geometrías complejas, conformación de la estructura, envolventes, y la interrelación de ambas, en materiales, sistemas constructivos y el software de diseño y modelado 3D que posibilitaron el despiece, modificación o retroalimentación de las soluciones constructivas de las propuestas planteadas. Análisis de elección de materiales, tipo de fabricación, en obra o desde lugares de fabricación especializados, para materializar los elementos proyectados (Baliński \& Januszkiewics, 2016).

8. Exposición y clasificación en 3 categorías, 8 casos de estudio acorde al tipo de producción de donde surgen; análisis del diseño general, el software de representación y los procesos de modelado; las dimensiones y características de la estructura como: nodos, segmentos, ángulos de variación y los materiales empleados en las envolventes (Golański, 2018).

\section{DOMINIO DE CONOCIMIENTO, DIMENSIONES DE APRENDIZAJE Y HERRAMIENTAS DE DISEÑO}

En el material revisado, se puede reconocer el dominio de pensamiento de diseño computacional y modelado. Entre los principales softwares de diseño se identifican: Rhinoceros Grasshopper y la plataforma Kangaroo, plugin Scan-and-Solve, Autodesk Revit R_, Autodesk Fusion360 R_).

En el caso del tipo de fabricación digital, el sistema más usado es el corte CNC, en material MDF. En el caso de la fabricación aditiva, se presentan el uso de materiales como el metal, concreto, y compuestos a base de tierra. Como retos particulares, estos se presentan sobre los materiales elegidos, caso de varillas, dobles sin ponerse en riesgo de fractura, por lo que se plantea encontrar un material avanzado que refuerce los elementos que conforman la estructura. En las dimensiones de aprendizaje de comportamiento de diseño asociativo, el usuario puede manejar y modificar la relación entre los objetos, empleando expresiones matemáticas que describen el proceso generativo de las relaciones numéricas o gráficas y en la retroalimentación entre el diseño, material y el diseñador. Esto representa un reto al momento de calibrar las cualidades físicas de los materiales y la comprensión de los materiales dentro del sistema digital.

\section{RESULTADOS: CONOCIMIENTO INSTRUMENTAL Y ANÁLISIS DE CASOS}

\section{CONOCIMIENTO INSTRUMENTAL}

1. Cambiando los parámetros correspondientes a las restricciones de los materiales como la resistencia y deformación para realizar la simulación, se puede verificar la viabilidad de construir o producir físicamente lo modelado digitalmente, aquí se evidencia lo necesario de integrar los aspectos tectónicos: la solución estructural del diseño planteado y el material elegido para lograr un resultado exitoso (Huang, Yan, Luo, \& Li, 2016). 
2. Las herramientas digitales permiten una creación creativa desde un proceso generativo de diseño conceptual no estructurado, donde la fabricación digital generalmente expresa y entrelaza la relación estructural de la etapa conceptual con el proceso de fabricación, reduciendo la distancia entre las etapas de diseño y de realización (Indrawan, Purwoko, \& P. Utomo, 2019).

3. La relación entre las herramientas digitales y la propuesta de formas, diferentes y personalizadas, modifican los pensamientos de diseño, las herramientas y los materiales determinan el proceso en la transición de lo digital a lo físico, solucionando las formas y el número de componentes, integrando en los modelos físicos y virtuales la posibilidad de solucionar los problemas estéticos, estructurales y de fabricación. Los autores alertan que estas herramientas pueden generar proyectos estéticamente atractivos, pero en muchos casos se puede caer en suposiciones inadecuadas o excesivas para su empleo en el mundo real; sin embargo, podrían representar una manera de mejorar la industria de la construcción haciéndola sustentable y colaborativa entre los diferentes actores, arquitectos, ingenieros, contratistas y artesanos (Rabite De Almeida, Lima, \& Martins Borges, 2019).

4. En los modelos paramétricos se adapta las variaciones de: material (diámetro, solides, nodos, espaciado, rectitud capacidad estructural) uniones (tipo, número y ángulo de las intersecciones, ángulos de rotación, flexibilidad) y diseño (General, forma, ancho, puntos de carga, capacidad estructural del ensamblaje); permitiendo una retroalimentación entre el diseño, material y el diseñador; pero a su vez representa un reto al momento de calibrar las cualidades físicas de los materiales y la comprensión de los materiales dentro del sistema digital (MacDonald, Hauptman, \& Schumann, 2019).

5. Conocimiento operativo cualitativo sobre las capacidades, los parámetros dimensionales y formales y las configuraciones de impresión 3D alcanzables de acuerdo al diseño del material, el brazo robótico y proceso de extrusión utilizados; prueba sobre los límites de aplicabilidad y oportunidades de diseño que ofrece la cob impresa en 3D. Presentando el cambio de un sistema de masa a un sistema basado en filamentos generando una flexibilidad de diseño y configuraciones constructivas, reconocimiento de las interacciones emergentes incorporadas con la materia. (Veliz Reyes, y otros, 2019)

\section{ANALISIS DE CASOS}

1. Las geometrías generales de las estructuras curvas resultan en formas complejas que deben considerar el desempeño estructural, los métodos de fabricación el material y los atributos arquitectónicos como parte de sus soluciones. Los resultados varían dependiendo de los sistemas de fabricación locales, la tecnología constructiva, las habilidades de construcción disponibles en la zona, así como del presupuesto disponible. En el caso del análisis del edificio, para el diseño de las geometrías complejas encuentran necesario subdividir la geometría en pequeñas figuras construibles (Yu, Hahm, \& Bhooshan, 2014).

2. Diversos problemas técnicos que representa el modelado para construcción de estructuras curvilíneas y como estos son un factor de cambio de las ideas iniciales o de bosquejo; así como la necesidad de desarrollar o emplear nuevos materiales que permitan la continuidad de los sistemas con la envolvente, ya que los "viejos" materiales y técnicas deben resolver nuevas geometrías curvilíneas (Baliński \& Januszkiewics, 2016).

3. Las herramientas digitales y de diseño paramétrico permiten relacionar las características de los materiales con las restricciones de función, fuerza y capacidad de carga de los elementos estructurales, desarrollándolos y "ajustándolos" con formas naturales que operan como un todo. El uso de tecnologías computacionales permite diseñar y calcular a detalle y precisión las estructuras (madera laminada), solucionando la forma que ofrece máxima eficiencia estructural, lográndolo mediante la interconexión entre el diseño paramétrico, modelado/simulación 3D y la fabricación digital con $\mathrm{CNC}$, lo que replantea un nuevo paradigma tectónico en la realización de arquitecturas no-estándar elaboradas con madera laminar (Golański, 2018).

\section{CONCLUSIONES Y RECOMENDACIONES DE LAS INVESTIGACIONES}

Entre las recomendaciones de las investigaciones revisadas, destacan:

a. La contribución a un pensamiento tectónico donde el material, la forma y la estructura son parte inherente del pensamiento arquitectónico, mediante la integración de los modelos físicos y virtuales con las soluciones a los problemas estéticos, estructurales y de fabricación.

b. Las soluciones optimizadas de la estructura que corresponden a un desarrollo de diseño ideal para su producción/construcción, los resultados varían, dependiendo de los sistemas de fabricación locales, la tecnología constructiva, las habilidades de construcción disponibles en la zona, así como del presupuesto del que se disponga. En algunos casos, como en los de las construcciones de geometrías complejas, es necesario subdividir la geometría en pequeñas figuras construibles.

c. La posibilidad de construir elementos complejos de forma rápida y con menos material, resolución a problemas técnicos concretos con elementos variables y ajustables, ideal de la técnica para reproducir elementos descatalogados o deteriorados. Estos sistemas de fabricación digital integran la esfera de diseño con la construcción, ya que representan una relación indisociable que inspira el diseño desde sus inicios.

d. La necesidad de desarrollar o emplear nuevos materiales que permitan la continuidad de los sistemas estructurales, con la envolvente.

e. El análisis de las características de los materiales con las restricciones de función, fuerza y capacidad de carga de los elementos estructurales, diseñados y calculados a detalle y precisión, solucionando la forma que ofrece máxima eficiencia estructural, lográndolo mediante la interconexión 


\section{DISCUSIÓN Y CONCLUSIONES: ENFOQUES DE TECTÓNICAS DIGITALES}

En resumen, podemos observar que tanto las investigaciones instrumentales como las documentales que se desarrollan a nivel internacional, tienen una mirada hacia el desarrollo de los aspectos que pueden incluirse o desarrollarse dentro de las tectónicas digitales. Como principales concepciones a resaltar mencionaremos las siguientes:

En el articulo de Yu, Hahm, \& Bhooshan (2014) los autores emplean este concepto para englobar el desarrollo y la búsqueda de la forma y los métodos de fabricación interdisciplinares que utilizan metodología computacional e integran de principio a fin las restricciones reales de la industria de la construcción en el actual proceso de diseño

Baliński y Januszkiewics (2016) lo plantean como una metodología que integra el uso de software de diseño digital con los métodos tradicionales de construcción, integrando lo computacional y la fabricación digital como una parte fundamental del proceso de diseño, ya que permiten comprender a detalle el comportamiento de las superficies que reciben la carga, permitiendo generar nuevas formas arquitectónicas.

Con esta idea de tectónicas digitales, Golański (2018). busca hacer referencia no solo a la realización precisa de la arquitectura, encontrando su solución como una habilidad técnica/digital, sino como una posibilidad de diseño/fabricación, que permite mediante las herramientas digitales, representar los elementos esenciales de los materiales con las restricciones de función, fuerza y capacidad de carga de los elementos estructurales, desarrollándolos y "ajustándolos" con formas naturales que operan como un todo.

De acuerdo con Rabite De Almeida, Lima, \& Martins Borges (2019), se identifica la relación de la tectónica con el movimiento de diseño de las relaciones geométricas y espaciales utilizadas en combinación para la formación de detalles y componentes, basado en herramientas digitales, debido a las nuevas posibilidades que ofrecen las herramientas digitales con respecto a la forma en que se diseña, evalúa y se producen los diseños y que abordan este nuevo producir/pensar el proceso de diseño en la arquitectura con el uso combinado de software paramétrico computacional con variables y parámetros digitales aunado a los constructivos que permite conocer el potencial de los diferentes grados de combinación que resultan de estas tecnologías y que muestran una interrelación entre: arquitectura, ingeniería, computación, análisis físicos, diseño y construcción.

Veliz Reyes et. al (2019) resaltan los aspectos fundamentales que emergen con el uso de las tecnologías robóticas como las propiedades materiales, la forma de la producción y la representación del conocimiento, así como la tectónica emergente e interacción incorporada. No solo se reconoce el cambio en las configuraciones estructurales y formales con la rotica sino las nuevas oportunidades tectónicas y de diseño para las estructuras resultantes de estos procesos de fabricación digital, con lo que se evidencian estudios críticos que delineen no solo el potencial de la arquitectura y sus lenguajes emergentes sino de igual manera sus cualidades tectónicas.

Resumiendo lo anterior podemos concluir que el concepto de tectónica digital, ya sea desarrollado desde la parte instrumental o teórica, podría presentarse como: la integración mediante las herramientas digitales de los aspectos formales, estructurales y de los materiales proyectados desde las etapas de diseño conceptual, y que integran en la lógica constructiva, los sistemas estructurales, constructivos, envolventes y de los materiales, planteando componentes tectónicos de fabricación digital, acorde al contexto socio-cultural de nuestros tiempos.

Posterior a la labor investigativa alrededor del tema de las tecnologías digitales y el componente tectónico de la arquitectura, el análisis de los autores, enfoques y metodologías nos permite determinar que este tema, es actual y se encuentra siendo investigado con avidez alrededor del mundo, de manera satisfactoria, lo que permite dilucidar diversas líneas de generación de conocimiento en el campo de la arquitectura, retomando el planteamiento de lo que significan conceptos históricos que se mantienen en constante evolución y revisión de acuerdo a los tiempos en los cuales se enmarca.

Contrariamente a las advertencias de Picon (2004), el avance instrumental, no solo del diseño asistido por computadora sino de las tecnologías de fabricación digital nos permiten discrepar (y él consigo mismo) y ver el largo camino que ha recorrido la arquitectura en los últimos años, mostrándonos y permitiéndonos coincidir con pensamiento de Picon (2019) que en el escenario contemporáneo de la arquitectura, la fabricación digital expresa un cambio de la comprensión de la materialidad y de nosotros mismos, ya que nos permite extraer información de la materia: separándola, almacenándola y posteriormente reinsertándola al mundo físico por diversos medios; fusionando información y materia, planteado nuevas objetividades y entendimientos de la naturaleza de la materia.

Con el desarrollo de estas nuevas posibilidades y perspectivas de integrar diseño, análisis, fabricación/ montaje que redefinen las relaciones entre la ideación y fabricación como un aspecto de la tekne contemporánea, es necesaria la discusión de esta influencia sobre los aspectos tectónicos particulares de cada tipo de fabricación digital, ya que se nos presenta como una realidad actual, cercana y en constante evolución, que nos exige comprender la práctica profesional arquitectónica desde aspectos interdisciplinares que buscan no solo dominar los aspectos técnicos.

En general, es posible que nos encontramos ante un paradigma "emergente" en la tectónica de fabricación digital, donde el diseño arquitectónico se ve renovado por el interés en la producción mediante herramientas y pensamientos de diseño enfocados hacia lo constructivodigital, que se enmarcan en procesos abiertos de elaboración y negociación del material, que también implica el análisis de las propiedades y los modos de producción y representación del conocimiento de fabricación digital, empleados en cada propuesta contemporánea que relacione estas dos esferas, que en 
este trabajo se ha definido como tectónicas de fabricación digital.

Las investigaciones que relacionan los aspectos tectónicos y constructivos con aspectos teóricos o instrumentales enfocados a las tecnologías de fabricación digital son variadas. Este trabajo busca relacionar los aspectos de las tectónicas de fabricación digital con los hechos arquitectónicos escala 1:1 fabricados aditivamente mediante una metodología de análisis de casos de estudio que nos ayuda a comprender cómo las tecnologías digitales modifican la forma de diseñar y fabricar la arquitectura actualmente.

\section{AGRADECIMIENTOS}

Los autores (becarios) agradecen al CONACYT y a la Maestría en Arquitectura de la Facultad de Arquitectura Xalapa de la Universidad Veracruzana perteneciente al PNPC-CONACYT, que acoge la investigación previamente mencionada, por su invaluable apoyo en la realización de esta investigación, así como a profesores, asesores, compañeros y familia sin los cuales no habría sido posible su desarrollo. SDG.

\section{REFERENCIAS}

Andersson, K., \& Kirkegaard. (2006). A discussion of the term digital tectonics. In (Ed.), A. ALI, \& C. Brebbia, Digital Architecture and Construction (pp. 29-39). Wessex: WIT Press.

Baliński, G., \& Januszkiewics, K. (2016). Digital Tectonic Design as a New Approach to Architecural Design Methodology. Procedia Engineering 161, 1504 - 1508.

Frampton, K. (1999). Estudios sobre cultura tectónica. Poéticas de la construcción en la Arquitectura de los siglos XIX Y XX. Madrid: Ediciones Akal, S.A.

Golański, M. (2018, Febrero). Digital tectonics and dynamics in designing of wooden. 11th Conference on Advanced Building Skins (pp. 759-769). Bern: Recuperado de:https://www.researchgate.net/publication/32322182 4_Digital_tectonics_and_dynamics_in_designing_of_w ooden architecture envelopes.

Huang, W., Yan, D., Luo, P., \& Li, X. (2016). Digital design and construction of a weaving structure. Conference: 8 th International Conference on Fiber-Reinforced Polymer (FRP) Composites in Civil.

Indrawan, S. E., Purwoko, G. H., \& P. Utomo, T. N. (2019). Reparametrizing Tectonics Perception on Planar Material-Design. International Journal of Creative and Arts Studies. Volume 6 Number 1, p-ISSN 2339-191X, e-ISSN 2406-9760, 69-77.

Iwamoto, L. (2009). Digital fabrications: architectural and material techniques. New York: Princeton Architectural Press.
Kolarevik, B. (2009). Architecture in the Digital Age. Design and Manufacturing. E-book. London- New York: Taylor \& Francis Group by Spon Press.

Liu, Y.-T., \& Lim, C.-K. (2006). New tectonics: a preliminary framework involving classic and digital thinking. Design Studies 27. Elsevier Ltd., 267-307.

MacDonald, K., Hauptman, J., \& Schumann, K. (2019). Digital Fabrication of Standard less Materials. Association for Computer Aided Design in Architecture. Ubiquity and Autonomy (pp. 266-275). Austin Texas: ACADIA.

Oxman, N., \& Rosenberg, J. L. (2007). Material-based Design Computation An Inquiry into Digital Simulation of Physical Material Properties as Design Generators. International Journal of Architectural Computing vol. 5 no. 1, 26-44.

Oxman, R. (2009). Digital Tectonics as a Morphogenetic Process. Proceedings of the International Association for Shell and Spatial Structures (IASS) Symposium , 938-948.

Oxman, R. (2012). Informed tectonics in material-based design. Design Studies 33. Elsevier Ltd , 427-455.

Picon, A. (2004). La arquitectura y lo virtual, hacia una nueva materialidad. In L. Ortega, La digitalización toma el mando. España: Gustavo Gilli.

Picon, A. (2019). Digital Fabrication, Between Disruption and Nostalgia: Notes on the Nature of Knowledge in Digital Architecture. DOI: 10.4324/9780429506338-23.

Rabite De Almeida, C. A., Lima, F., \& Martins Borges, M. (2019, Junio). Tectônicas Digitais: a (in) tangibilidade no processo de projeto em arquitetura. REVISTA DESIGN \& TECNOLOGIA, Vol. 09, No. 18, 01-21.

Real Academia, L. E. (2001). Tectónica. Retrieved Diciembre 2019 , from Diccionario de la Lengua Española ( $23^{\circ} \mathrm{ed}$.) Recuperado https://dle.rae.es/tect\%C3\%B3nico\#otras

Schumacher, P. (2017). Tectonism in Architecture, Design and Fashion: Innovations in Digital Fabrication as Stylistic Drivers. Architectural Design. 3D-Printed Body Architecture. November/December 06/Vol 87/2017, Recuperado de:DOI: 10.1002/ad.2245.

Sekler, E. (1965). Structure, construction \& tectonics. In G. K. (Editor), Structure in Art and in Science (pp. 89-95). New York: George Braziller, Inc.

Veliz Reyes, A., Jabi, W., Gomaa, M., Chatzivasileiadi, A., Ahmad, L., \& Mario Wardhana, N. (2019). Negotiated matter: a robotic exploration of craftdriven innovation. Architectural Science Review Vol. 62, 398-408.

Yu, S., Hahm, S., \& Bhooshan, S. (2014). Performance driven selfsupporting curved surface morphologies and tectonic in current practice. Next Generation Building. 